\title{
Transition to chaos in thermocapillary convection
}

\author{
Peng Zhu, Li Duan, Qi Kang* \\ Key Laboratory of Microgravity (National Microgravity Laboratory), Institute of Mechanics, Chinese Academy of Sciences, Beijing 100190, PR China
}

\section{A R T I C L E I N F O}

\section{Article history:}

Received 17 March 2012

Received in revised form 11 October 2012

Accepted 11 October 2012

Available online 17 November 2012

\section{Keywords:}

Thermocapillary convection

Transition to chaos

Critical condition

Transition route

\begin{abstract}
A B S T R A C T
We present experiments on transition to chaos in thermocapillary convection in a rectangular pool of silicone oil. The applied temperature difference between the two sidewalls is adjusted in the range of $0-43{ }^{\circ} \mathrm{C}$ to observe various dynamic states. The applied temperature gradient along the fluid-gas interface drives shear flow along the free surface from hot to cold and a back flow in the underlying layer. With the increase of the temperature gradient, the thermocapillary convection will transit from steady flow to regularly oscillatory flow, and finally to chaos. A temperature measurement system, which consists of thermocouple, voltmeter and data-acquiring computer, is used to record the temperature of the liquid dynamically. In order to identify the different dynamic regimes from steady flow to chaos, fast Fourier transform and fractal theory are used to analyze the experimental data. The critical conditions for transition have been obtained and discussed by non-dimensional analysis. The quasi-periodic route and Feigenbaum route were observed for different experimental conditions, and the relationship between oscillatory frequency and Marangoni number $M a$ has been discussed.
\end{abstract}

(c) 2012 Elsevier Ltd. All rights reserved.

\section{Introduction}

Thermocapillary convection is driven through the surface tension gradient, which is produced by a temperature gradient along the free surface. When the applied temperature gradient is increased, the convection will transit from steady flow to a sequence of instabilities, and finally to chaos or turbulence. The transition to chaos in thermocapillary convection has been of interest for its practical and theoretical value [1,2]. Critical conditions and transition routes are two important aspects of transition to chaos.

A large amount of researches has been done on the critical conditions and instabilities at threshold. Smith and Davis [3,4] performed a linear stability analysis of thermocapillary instability. When the free surface is assumed to be flat and non-deformable, they found two types of thermocapillary instabilities: stationary longitudinal rolls and hydrothermal waves. When the free surface is thought to be deformable, the instability of surface wave was obtained. And then the theoretical analysis of the thermocapillary instabilities have been made up by Parmentier [5] for the consideration of buoyant effect, Mercier and Normand [6] for the introduction of heat exchange to the atmosphere and Kuhlmann [7] for three-dimensional flow. Experiments on thermocapillary instability have been conducted by Riley and Neitzel [8]. They have observed two kinds of instabilities through instantaneous thermograph in a rectangular pool. For small Bond number, the ori-

\footnotetext{
* Corresponding author. Tel.: +86 1082544112 .

E-mail address: kq@imech.ac.cn (Q. Kang).
}

ginal steady unicellular flow transits to hydrothermal waves. For large Bond number, it transits to steady multicellular flow first, and then to oscillating multicellular flow, which features steady multicellular structures near the hot wall and a pair of oblique waves near the cold wall. Not only the hydrothermal waves, but Burguete [9] has also observed the instability of stationary rolls for deep liquid layer by means of shadowgraph images. Critical temperature differences for several aspect ratios have been collected.

Transition routes from laminar to turbulence regime in Rayleigh-Benard convection [10-13] and Benard-Marangoni convection [14] have been studied extensively both experimentally and numerically. While the temperature-gradient directions in the convections mentioned above are in vertical, transition routes in thermocapillary convection applied with horizontal temperature-gradient have been much less investigated. Bucchignani and Mansutti $[15,16]$ reported the numerical results of bifurcation pattern of thermocapillary convection. They used the Rayleigh number $R a$ as the bifurcation parameter. When $R a=4.25 \mathrm{e} 8$, an unsteady periodic flow with a fundamental frequency was obtained. Then an increase of $R a$ at $4.3 \mathrm{e} 8$ leads to the second Hopf bifurcation from the periodic flow to a quasi-periodic regime with two incommensurate frequencies. At $R a=5 \mathrm{e} 8$, the presence of a quasi-periodic regime with three incommensurate frequencies has been observed. And then it develops into chaotic flow. This kind of bifurcation sequence with the characteristic of quasi-periodic bifurcation is named Ruelle-Takens-Newhouse route, which is one of three well-known routes to chaos. [2] Besides the Ruelle-Takens-Newhouse route, the Feigenbaum 


\section{Nomenclature}

$h \quad$ depth of the liquid layer, $m$

$t$ time, $s$

$v \quad$ dynamic viscosity, Pa s

$\gamma \quad$ kinetic viscosity, $\mathrm{m}^{2} \mathrm{~s}^{-1}$

$L_{x} \quad$ stream-wise domain length, $\mathrm{m}$

g gravitational acceleration, $\mathrm{m} \mathrm{s}^{-2}$

$T$ temperature of the measurement point, ${ }^{\circ} \mathrm{C}$

$\triangle T$ applied temperature difference between the two sidewalls, ${ }^{\circ} \mathrm{C}$ $f_{1} \quad$ the first fundamental frequency, $\mathrm{Hz}$

$f_{2}$ the second fundamental frequency, $\mathrm{Hz}$

Greek symbols

$\beta \quad$ thermal expansion coefficient, $1 /{ }^{\circ} \mathrm{C}$

$\kappa \quad$ thermal diffusivity, $\mathrm{m}^{2} \mathrm{~s}^{-1}$

$\rho \quad$ density, $\mathrm{kg} \mathrm{m}^{-3}$

$\sigma \quad$ surface tension, $\mathrm{N} \mathrm{m}^{-1}$ route is characterized by period-doubling bifurcation, and the Pomeau-Manneville route by intermittent appearance of periodic and chaotic phases. The other two transition routes were not reported in Mansutti's study. There is also a lack of experimental results of transition routes in thermocapillary convection in rectangular pool.

Study on transition to chaos in thermocapillary convection is beneficial to explore the mechanism of thermocapillary instability. And experiments on transition routes of thermocapillary convection in rectangular pool need to be studied to make up the lack. The aim of the present research work is to obtain critical conditions for the transition, and to identify transition routes to chaos. To achieve the goal, we designed a temperature measurement system with high resolution to record the temperature of the liquid dynamically. In order to identify the transition routes from steady flow to chaos, fast Fourier transform and fractal theory are used to analyze the experimental data.

The paper is organized as follows: Section 2 gives a brief description of the thermocapillary problem and the analysis method to investigate it. In Section 3, we first study the critical conditions for the transition; our results are discussed and compared with others. Then, transition routes to chaos are studied for two experimental conditions; transitions in the two conditions follow the quasi-periodic route and Feigenbaum route, which is identified experimentally in thermocapillary convection in rectangular pool for the first time. Finally, the main findings are summarized in Section 4.

\section{Experimental setup and procedure}

In order to research on evolvement of temperature oscillation in our laboratory, we have constructed a buoyancy-thermocapillary convection system as shown in Fig. 1. The rectangle pool is made up of a right hot end with the thickness of $6 \mathrm{~mm}$ made of copper heated by an electrothermal film and a left cold end with the same thickness. The horizontal cross-section of the pool is $52 \times 36 \mathrm{~mm}$, and the height of the pool is $6 \mathrm{~mm}$. The front, rear and bottom side of the pool is made of optical glass $\mathrm{K} 9$ with the thickness of $6 \mathrm{~mm}$.
In our experiment, the working fluid is silicone oil of $0.65,1$, and 1.5 cSt whose Prandtl numbers are equal to 10,16 , and 25 respectively. The horizontal temperature gradient in the fluid layer will be established between the two copper walls. A DC electrical power is controlled by a temperature controller to work the electrothermal film to heat the hot end. The temperatures of the two copper walls are measured through two T-type thermocouples. With the increase of the temperature difference between the two copper walls, the convection in the fluid layer in the rectangle pool will transit from stable to unstable. The working fluid used in our experiments is silicone oil $0.65,1$, and $1.5 \mathrm{cSt}$, whose physical properties are given in Table 1.

To record the temperature evolvement in the fluid layer in a period of time, we have designed a temperature measurement system, which consists of a thermocouple, a voltmeter, and a personal computer. The sensor in the system is thermocouple, the diameter of whose wire is about $60 \mu \mathrm{m}$. The operating principle is shown in Fig. 2. Thermocouple transfers the temperature signal to voltage signal, which will be measured by the voltmeter. Finally, the temperature is calculated and is recorded by personal computer. During our experiment, the temperature magnitudes are recorded with the sampling rate of $5 \mathrm{~Hz}$.

To identify the different dynamic regimes during the transition to chaos, the fast Fourier transform is used to calculate the power spectrum of a dynamic variable. Periodic, quasi-periodic and period-doubling flows can be recognized from the power spectra. Chaotic flow is considered to occur, when a signal broadband develops in the power spectrum.

Fractal theory becomes more and more popular in the application of chaotic dynamic analysis. Chaotic time series can be represented with a fractal through reconstruction technique, which stems from the embedding theorem developed by Takens [17] and Sauer [18]. Fractal dimension $D$, one of the important and internal characteristics of a fractal, is usually estimated by calculating correlation dimension $D_{m}$. GP algorithm proposed by Grassberger and Procaccis [19] is a simple and reliable method to determine $D_{m}$. In the method, a correlation integral $C_{m}(r)$ is defined as

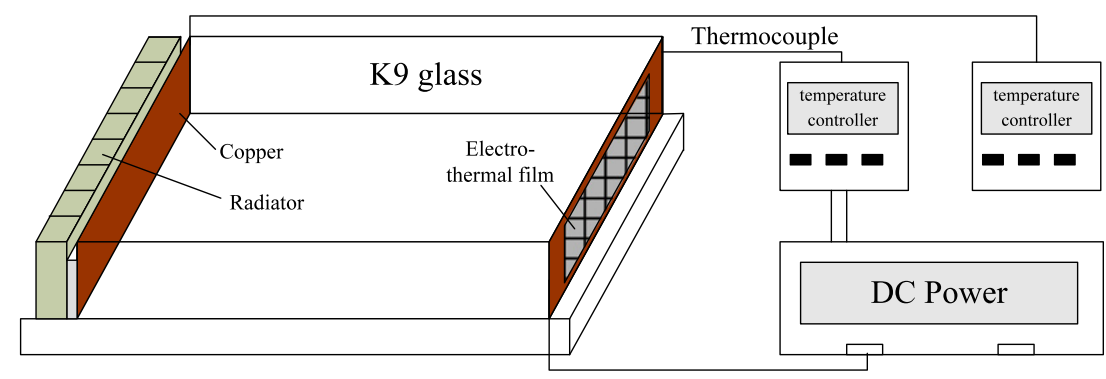

Fig. 1. Controlling system for buoyancy-thermocappilary convection. 
Table 1

Physical properties of silicone oil $0.65,1$ and $1.5 \mathrm{cSt}$ at $25^{\circ} \mathrm{C}$.

\begin{tabular}{|c|c|c|c|c|c|c|c|}
\hline Silicone oil (cSt) & $\gamma\left(\mathrm{m}^{2} \mathrm{~s}^{-1}\right)$ & $\rho\left(\mathrm{kg} \mathrm{m}^{-3}\right)$ & $\beta\left({ }^{\circ} \mathrm{C}^{-1}\right)$ & $\kappa\left(\mathrm{m}^{2} \mathrm{~s}^{-1}\right)$ & $\sigma\left(\mathrm{N} \mathrm{m}^{-1}\right)$ & $\partial \sigma / \partial T\left(\mathrm{~N} \mathrm{~m}^{-1}{ }^{\circ} \mathrm{C}^{-1}\right)$ & $\operatorname{Pr}=v / \kappa$ \\
\hline 0.65 & $6.5 e-7$ & 760 & $1.35 \mathrm{e}-3$ & $6.67 e-8$ & $1.59 \mathrm{e}-2$ & $-8.36 e-5$ & 10 \\
\hline 1 & $1 e-6$ & 818 & $1.29 \mathrm{e}-3$ & $6.19 e-8$ & $1.69 \mathrm{e}-2$ & $-7.55 e-5$ & 16 \\
\hline 1.5 & $1.5 \mathrm{e}-6$ & 852 & $1.27 \mathrm{e}-3$ & $5.95 e-8$ & $1.77 \mathrm{e}-2$ & $-7.35 e-5$ & 25 \\
\hline
\end{tabular}

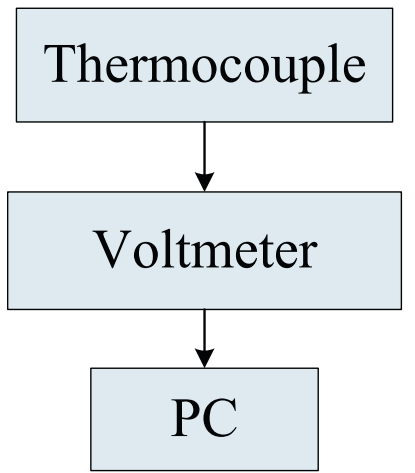

Fig. 2. Diagnostics techniques diagram of temperature measurement system.

$\mathrm{C}_{m}(r)=\frac{1}{l^{2}} \sum_{i=1}^{l} \sum_{j=1}^{l} \theta\left(r-\left\|X_{i}-X_{j}\right\|\right)$,

where $\theta$ is the Heaviside function, $X_{i}$ and $X_{j}$ are a pair of points in the reconstructed space of dimension $\mathrm{m}$, and $l$ is the total number of data points. Then the correlation dimension $D_{m}$ is defined as

$D_{m}=\lim _{r \rightarrow 0} \frac{\ln C_{m}(r)}{\ln r}$

So, to obtain the correlation dimension $D_{m}$, one should first plot the $\ln C_{m}(r)-\ln r$ curve through increasing the value of $m$ until the slope of the linear variation part is almost invariable. Then the convergent slope is regarded as the correlation dimension $D_{m}$. For deterministic chaos, there always exists a convergent slope as $D_{m}$, which is a real number larger than 2 . While it is random, the slope is always growing with $\mathrm{m}$. Therefore deterministic chaos and random signal can be distinguished through the existence of correlation dimension.

\section{Results and discussions}

A typical evolvement of transition to chaos is shown in Fig. 3, which is indicated by the temperature evolution in fluid layer. During the experimental process corresponding to Fig. 3, the applied temperature difference is controlled to be $11^{\circ} \mathrm{C}$ first, then it is increased to $30^{\circ} \mathrm{C}$ gradually from $t=600 \mathrm{~s}$ to $t=1600 \mathrm{~s}$, and then it is kept until $2350 \mathrm{~s}$. The added stable periodic state was measured with the same condition when the applied temperature difference is controlled to be $16{ }^{\circ} \mathrm{C}$ which is during transition process. When the fluid layer is applied with the small temperature difference $\triangle T=11^{\circ} \mathrm{C}$, the temperature at the measurement position in the fluid layer maintains a certain value; at this moment the flow in the rectangular pool is laminar. When the temperature difference reaches a critical value $\Delta T_{c}$, the temperature begins to oscillate periodically. When the temperature difference $\Delta T$ is increased further, the temperature oscillation will become more and more nonperiodic, and finally transit to chaos; meanwhile, the state of the convection becomes turbulent. In this study, our focuses are the critical conditions for temperature oscillation and the transition routes from the first threshold to chaos.

\subsection{Critical conditions}

The state of the flow in the rectangular pool can be reflected by the time history of the temperature at one measurement point in the fluid layer. Fig. 4(a) shows the time history of temperature when the fluid layer is applied with the temperature difference $\triangle T=6.3^{\circ} \mathrm{C}$. As we can see, the temperature varies randomly in a small range of $\pm 0.002{ }^{\circ} \mathrm{C}$. These temperature variations in the small range are just caused by environmental disturbance and measurement error. So the state of the flow is laminar, and it is below threshold. In Fig. 4(b), while the temperature difference reaches $9.9^{\circ} \mathrm{C}$, the measurement temperature oscillates periodically with the amplitude of $0.11^{\circ} \mathrm{C}$. So the convection has become oscillated, and it is above the first threshold.

Since the time histories of temperature below and above threshold can be measured as shown in Fig. 4, the critical

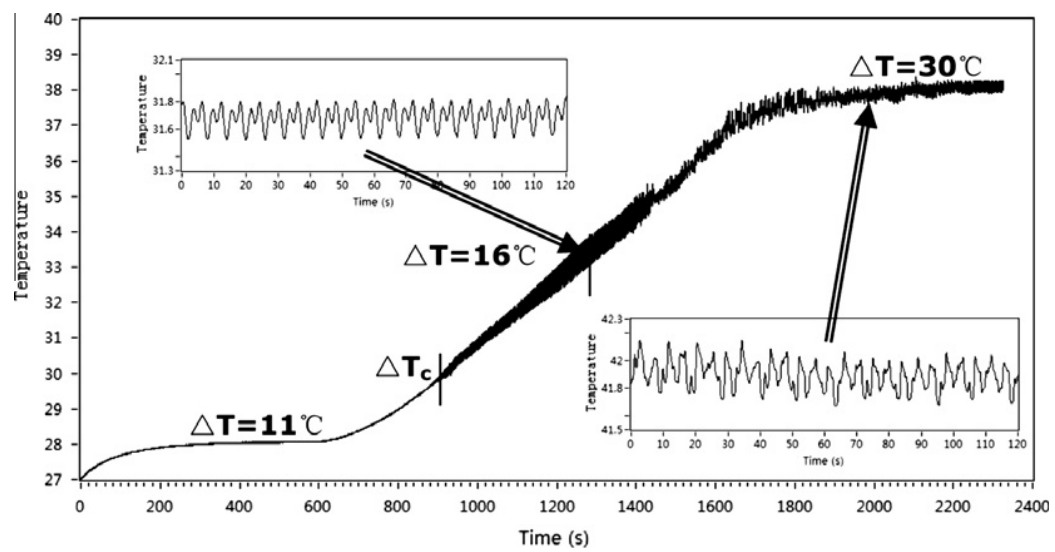

Fig. 3. Temperature evolution at one point of fluid layer in rectangular pool for silicone oil $1 \mathrm{cSt}$ with the depth of $3 \mathrm{~mm}$. 


\section{(a)}

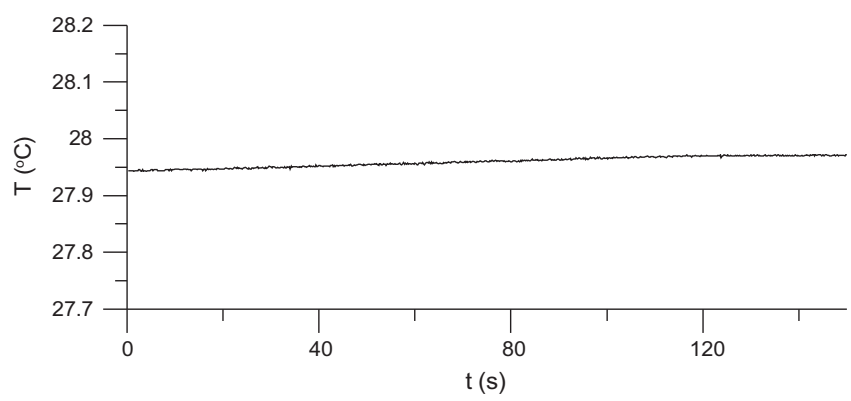

(b)

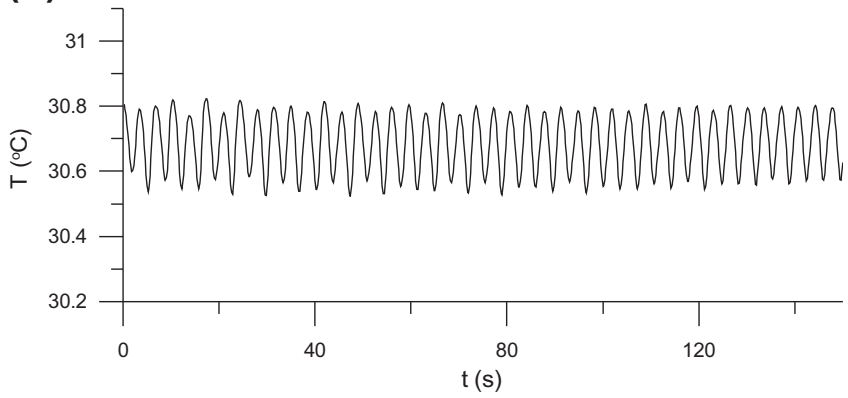

Fig. 4. Time history of temperature for silicone oil $1 \mathrm{cSt}$ with the depth of $2 \mathrm{~mm}$ : (a) $\triangle T=6.3^{\circ} \mathrm{C}$ (b) $\triangle T=9.9^{\circ} \mathrm{C}$.

temperature difference can be obtained by narrowing the range between the two different $\triangle T$. The critical temperature differences of oscillatory flow for different fluid depths are collected in Fig. 5. We have observed the temperature oscillation just for a certain range of layer depth; it is from $1.15 \mathrm{~mm}$ to $3.9 \mathrm{~mm}$ for silicone oil $0.65 \mathrm{cSt}$, and from $1.6 \mathrm{~mm}$ to $3.8 \mathrm{~mm}$ for silicone oil $1 \mathrm{cSt}$. For the fluid layer which is too thin, the mechanism of the destabilization is different because of the complication of the basic flow. Owing to the good immersion of experimental fluid with the wall of the rectangular pool, the fluid layer with larger depth is harder to be established. The dependence of the critical temperature difference on the depth of the fluid layer is consistent with the results obtained by Burguete [9]. The non-monotonic variation of $\triangle T_{c}$ with depth is thought to be caused by the different oscillation modes, which is of interest in our next step work. And the details of the critical conditions for oscillatory flow will be discussed below.

As we all known, the thermocapillary instability is basically driven by the tension gradient along the surface. So the incipience of

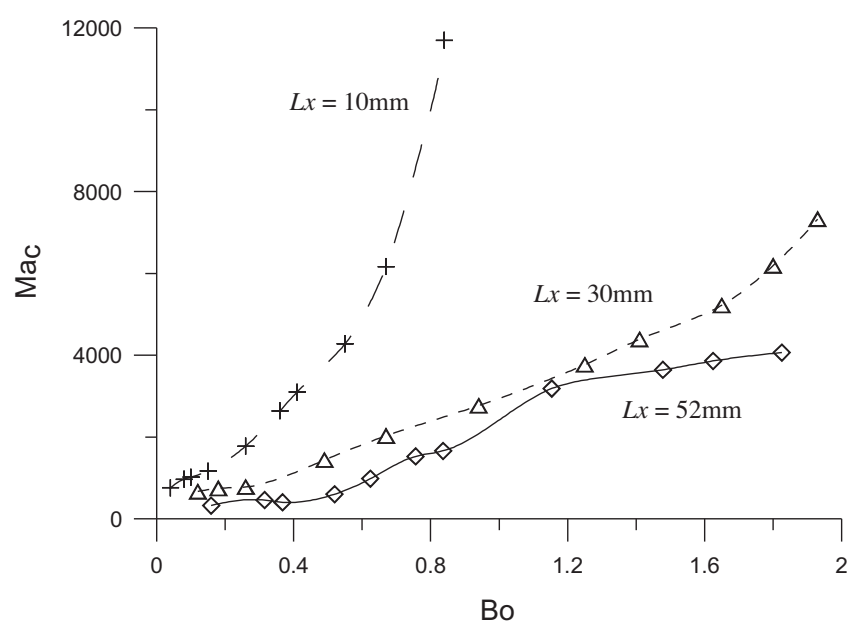

Fig. 6. Effect of Bond number and aspect ratio on the critical Marangoni number. $(+, \triangle)$ are experimental results obtained by Burguete [9]; $(\diamond)$ are experimental results obtained in this study.

the oscillatory flow can be indicated by the critical Marangoni number $M a_{c}$, defined as

$M a_{c}=(\partial \sigma / \partial T) \Delta T h^{2} /(\rho \gamma L \kappa)$

In our experiment on the ground, the effect of the buoyancy cannot be neglected. And the relative strength of buoyant forces to thermocapillary forces is measured by the Bond number, defined as

$B o=\rho g \beta h^{2} /(\partial \sigma / \partial T)$

In Fig. 6 the critical Marangoni numbers for liquid layer of $0.65 \mathrm{cSt}$ are shown. The results drawn in solid line are obtained in our study. The critical Marangoni numbers become larger with the increase of the Bond number. According to the definition of the Bond number, the effect of the buoyancy becomes larger with the increase of the Bond number. So we can infer that the enhancement of the buoyant effect in the whole flow can stabilize the convection; the inference is in agreement with the conclusion deduced from the experimental results measured by Schwabe and Scharmann [20]. Moreover, the results drawn in dashed line were collected by Burguete [9]. With comparison of the three groups of critical Marangoni numbers, the dependence of the critical condition on the aspect ratio $\Gamma=L_{x} / h$ is shown obviously. For larger aspect ratio of the fluid layer, thermocapillary convection in the rectangular pool
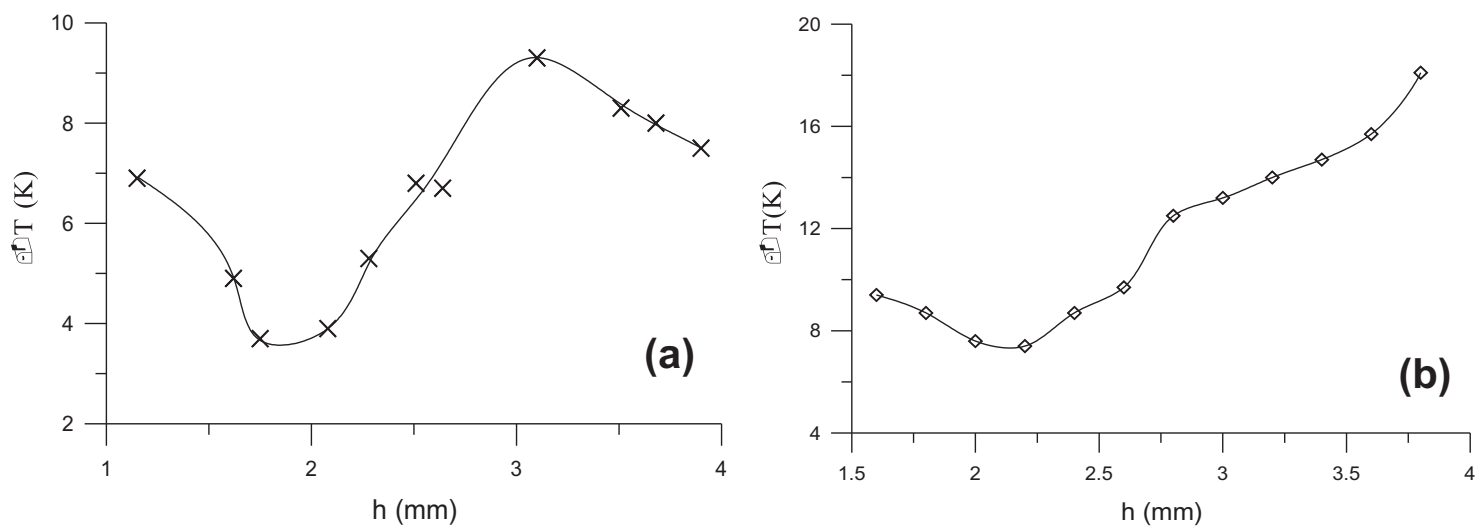

Fig. 5. Critical temperature differences vs. fluid depths: (a) $\operatorname{Pr}=10$, (b) $\operatorname{Pr}=16$. 
(a)

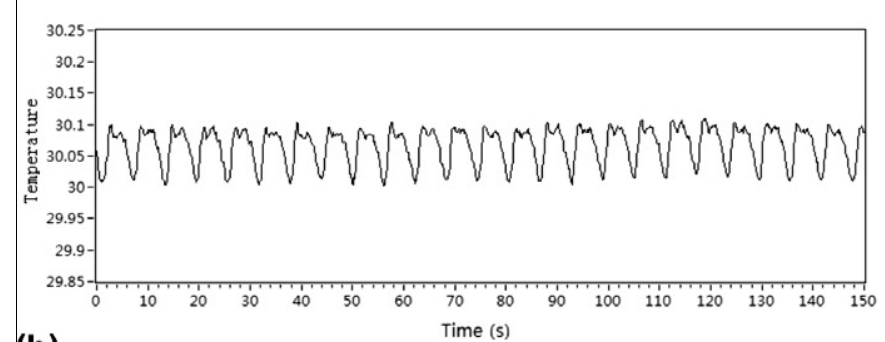

(b)

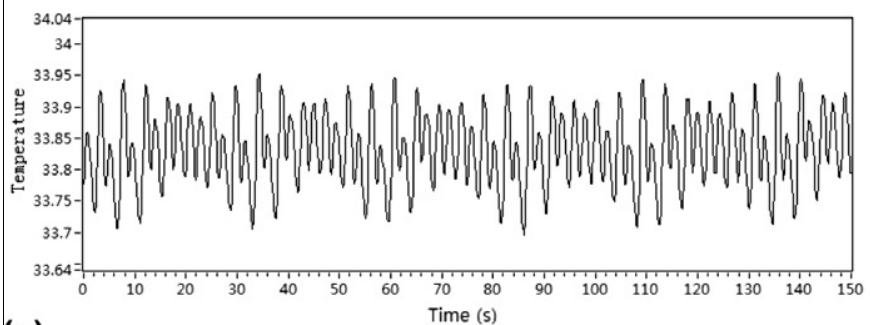

(c)

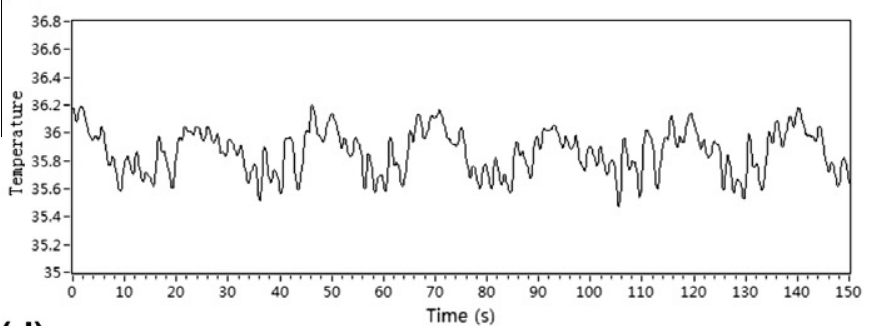

(d)

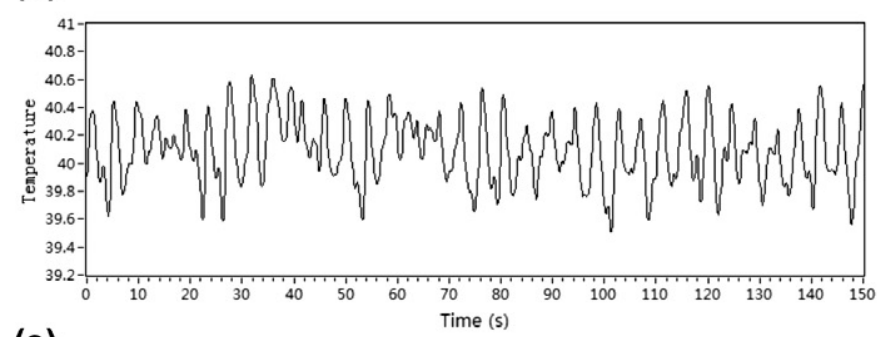

(e)

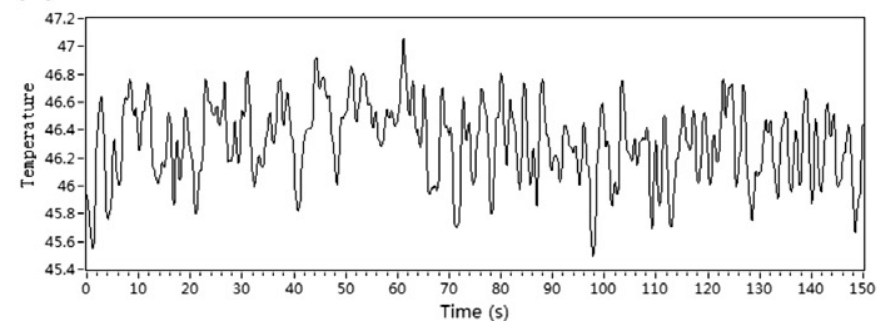

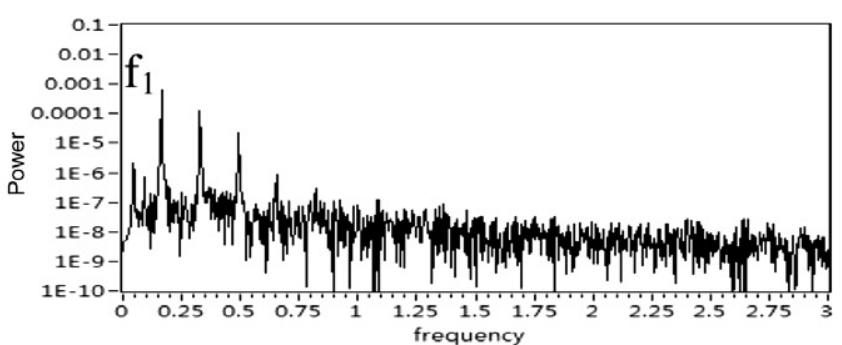
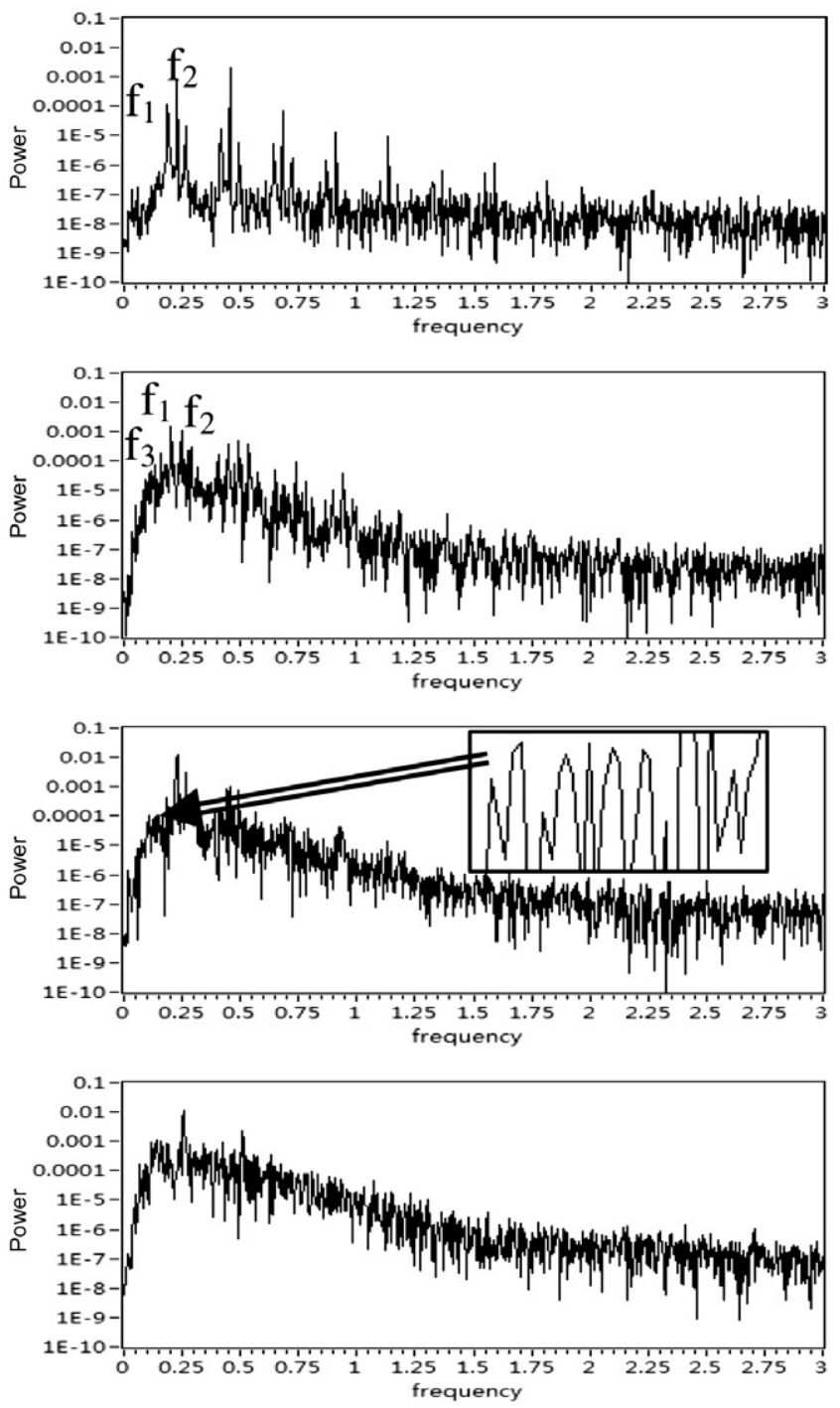

Fig. 7. Time history of temperature(left)and power spectra(right):(a) $\triangle T=15^{\circ} \mathrm{C}$; (b) $\triangle T=17.8^{\circ} \mathrm{C} ;(\mathrm{c}) \triangle T=19.8^{\circ} \mathrm{C} ;(\mathrm{d}) \triangle T=23.5^{\circ} \mathrm{C}$; (e) $\triangle T=29.3^{\circ} \mathrm{C}$.

is easier to destabilize to oscillatory flow. This conclusion is consistent with the results of experimental results for different aspect ratios by Burguete [9].

\subsection{Transition routes}

A sequence of temperature records and corresponding power spectra for various temperature differences is shown in Fig. 7. The evidence is obtained for silicone oil $1 \mathrm{cSt}$ with the depth of $3 \mathrm{~mm}$. Above the first threshold, thermocapillary flow first destabilizes to oscillate periodically, as can be seen from the time history of temperature and power spectra when $\triangle T=15^{\circ} \mathrm{C}$ in Fig. $7(\mathrm{a})$.
In the power spectrum, besides fundamental frequency $f_{1}=0.163 \mathrm{~Hz}$, there are also several harmonics at integral multiples of the fundamental. The flow becomes quasi-periodic when the temperature difference is increased to $17.8^{\circ} \mathrm{C}$, as shown in Fig. 7(b). A second fundamental frequency $f_{2}=0.226 \mathrm{~Hz}$ appears, and the former one slightly changes to $f_{1}=0.187 \mathrm{~Hz}$. And all the other peaks can be expressed by the linear combination of the two fundamental frequencies. When the temperature difference is increased to $19.8^{\circ} \mathrm{C}$, a third fundamental frequency appears, as can be seen in Fig. 7(c). In the power spectrum, all the peaks can be expressed by the linear combination of three incommensurate frequencies: $f_{1}=0.204 \mathrm{~Hz}, f_{2}=0.246 \mathrm{~Hz}, f_{3}=0.130 \mathrm{~Hz}$. As the 
(a)

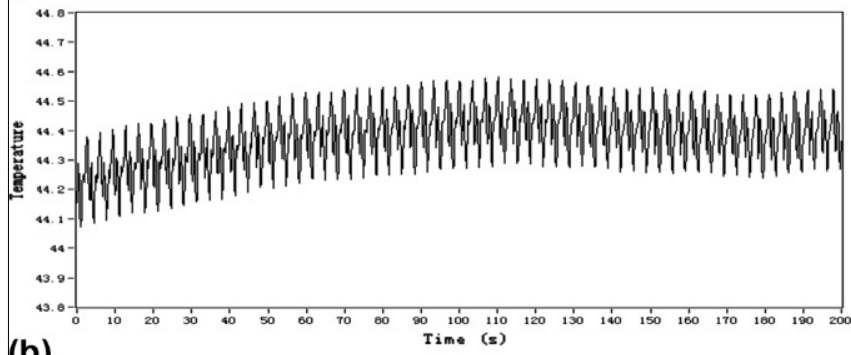

(b)

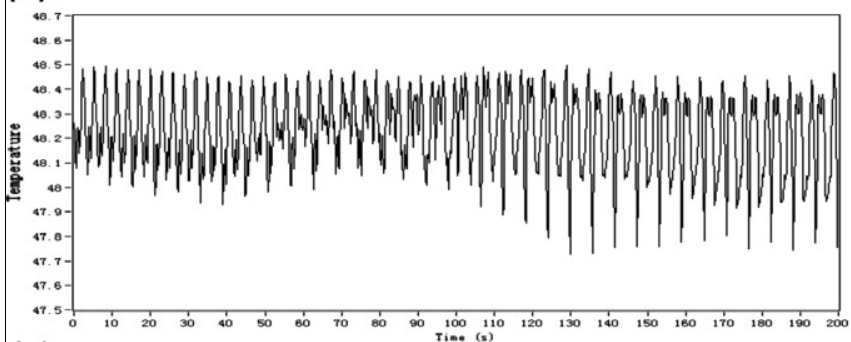

(c)

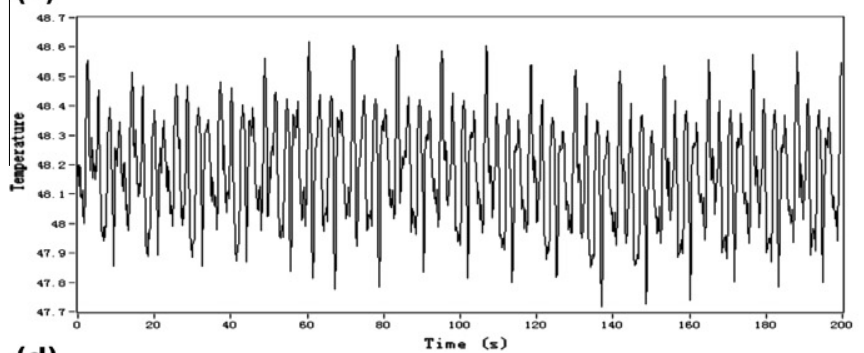

(d)

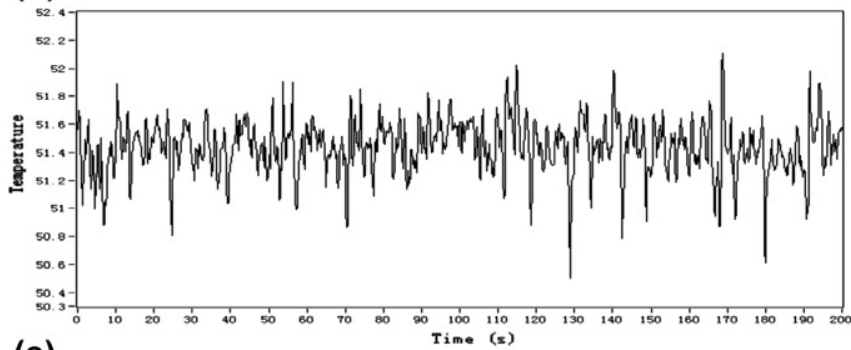

(e)

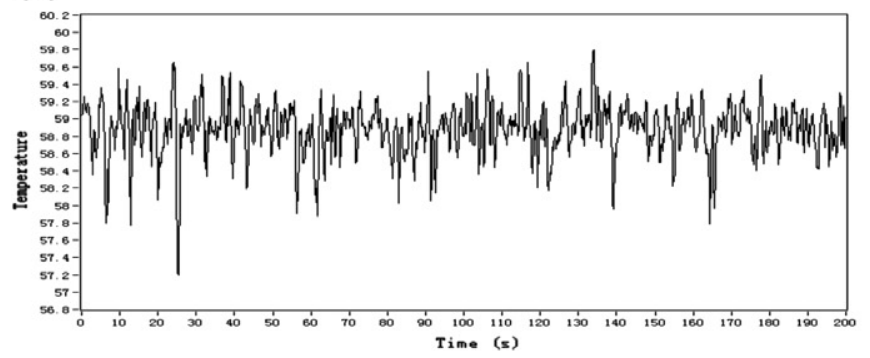

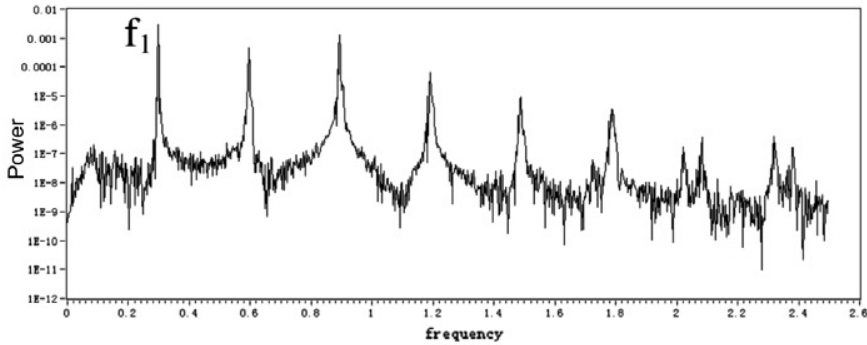
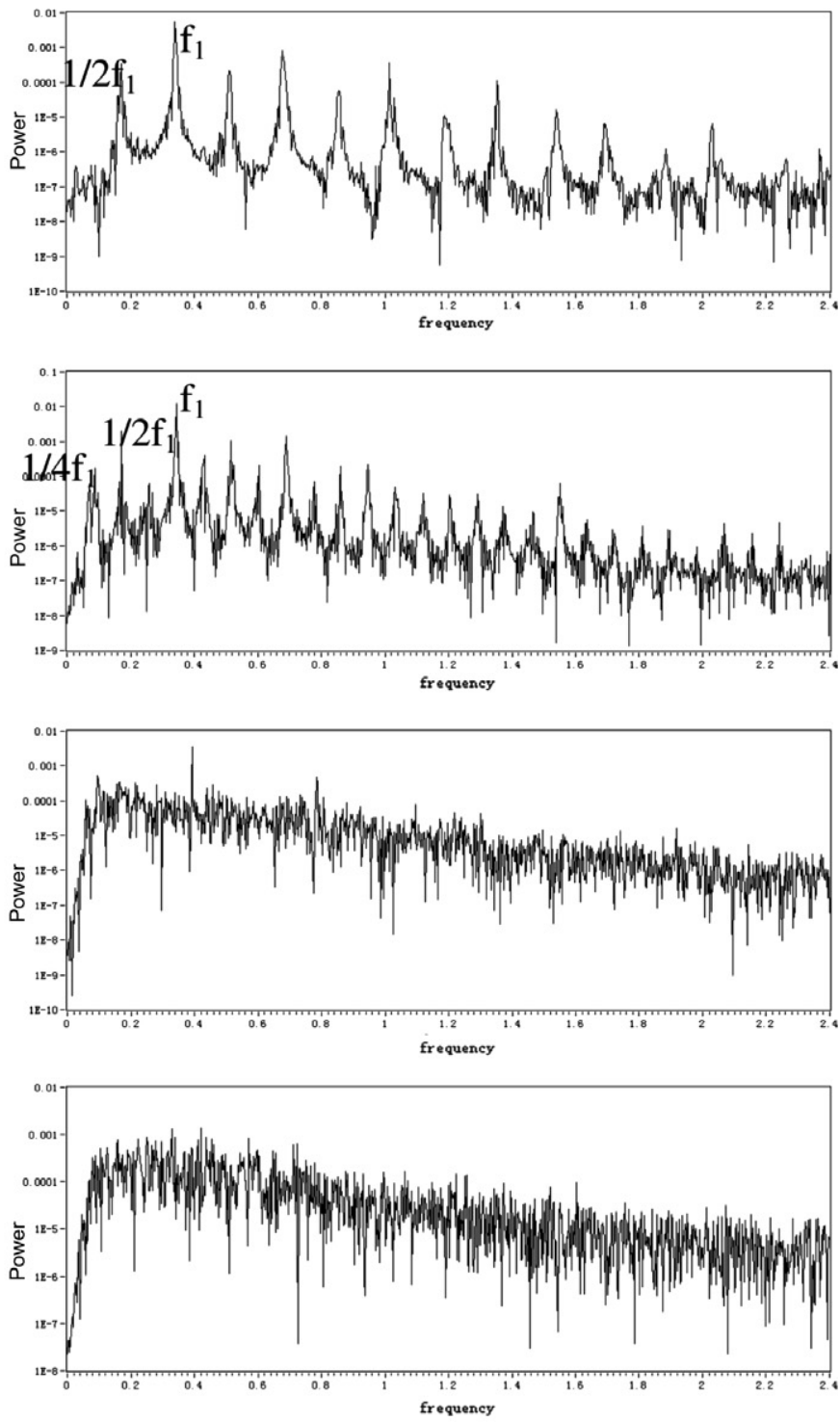

Fig. 8. Time history of temperature(left)and power spectra(right): (a) $\triangle T=28.1^{\circ} \mathrm{C}$; (b) $\triangle T=31.4^{\circ} \mathrm{C}$; (c) $\triangle T=32.3^{\circ} \mathrm{C}$; (d) $\triangle T=34.7^{\circ} \mathrm{C}$; (e) $\triangle T=43^{\circ} \mathrm{C}$.

temperature difference is increased further to $23.5^{\circ} \mathrm{C}$ (in Fig. $7(\mathrm{~d})$ ), the peaks in the power spectrum will become more; because of the overlap of the peaks, some broaden peaks will appear in local. Finally, the broaden peaks will develop to a broadband, as can be seen in Fig. 7(e). Rather than the broadband caused by the environmental noises, the signal broadband with the power of 3 order larger is due to the evolvement of flow oscillation. The appearance of the signal broadband reveals that the flow becomes chaotic. So we can summarize that, after the first threshold, the thermocapillary flow experience periodic state, quasi-periodic state, and finally become chaotic. During the quasi-periodic route, the temperature oscillation experiences quasi-periodic state with three incommensurate frequencies, which is necessary in the Ruelle-Takens-Newhouse route. But different from the Ruelle-Takens-Newhouse route, the oscillation doesn't transit to chaos immediately. When the temperature difference is increased further, the number of frequency increases gradually. With the development of the signal broadband, the temperature oscillation transits to chaos.

Besides the quasi-periodic route, we have also observed the Feigenbaum route for silicone oil $1.5 \mathrm{cSt}$ with the depth of $2 \mathrm{~mm}$, 

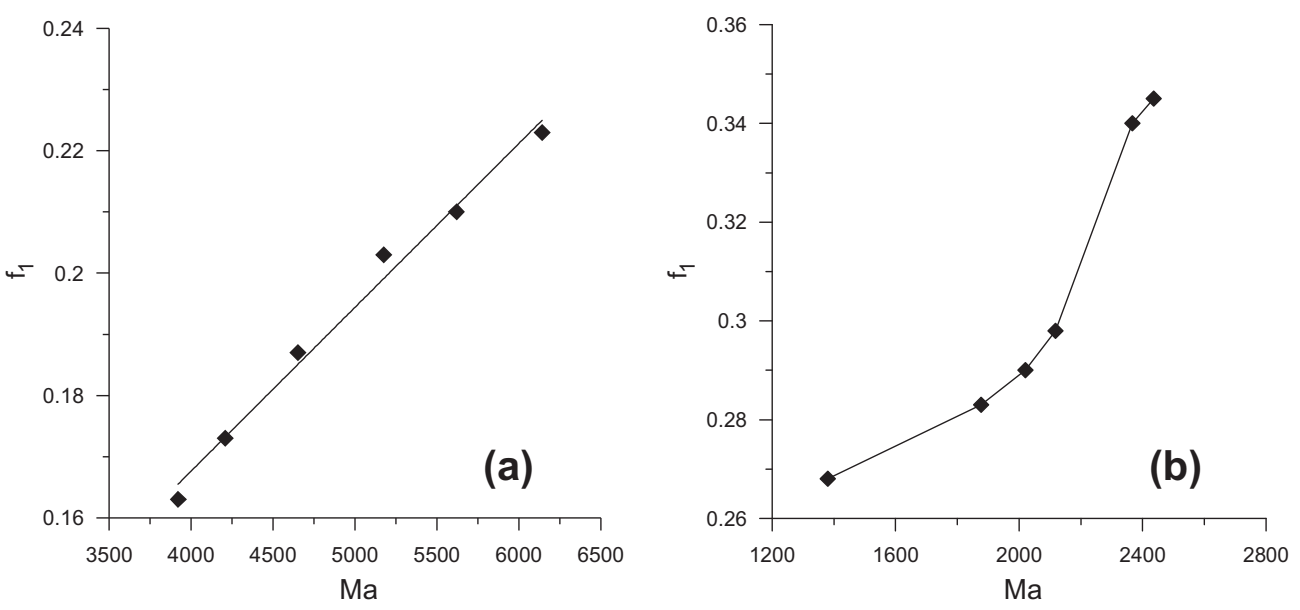

Fig. 9. Oscillatory frequencies vs. Marangoni numbers: (a) silicone oil $1 \mathrm{cSt}, h=3 \mathrm{~mm}$; (b) silicone oil $1.5 \mathrm{cSt}, h=2 \mathrm{~mm}$.
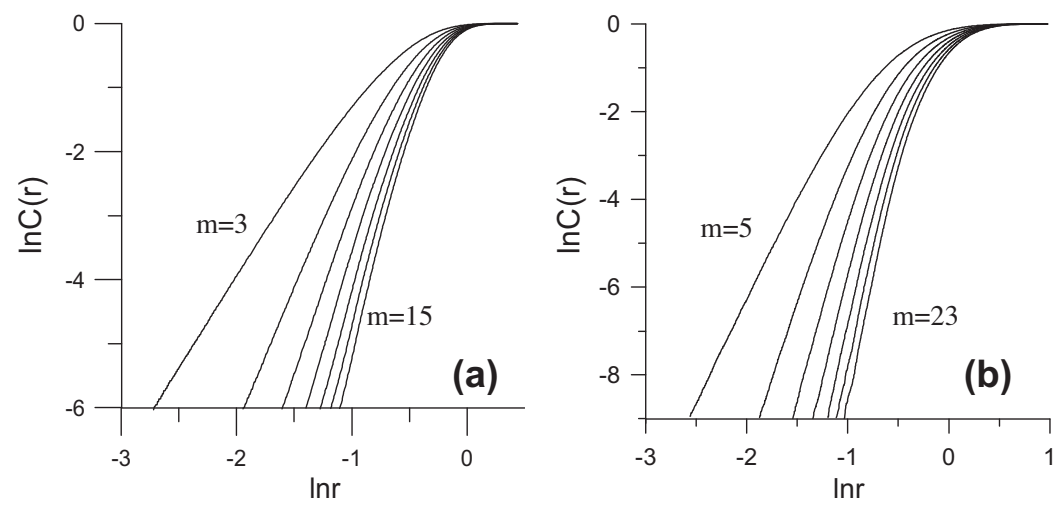

Fig. 10. Correlation dimension computation: (a) silicone oil $1 \mathrm{cSt}, \Delta T=29.3^{\circ} \mathrm{C}, m=3,5,7,9,11,13,15$; (a) silicone oil $1.5 \mathrm{cSt}, \Delta T=43{ }^{\circ} \mathrm{C}, m=5,8,11,14,17,20,23$.

which is characterized by period-doubling bifurcation. After the incipience of the oscillation, the flow still first becomes periodic state, whose power spectrum consists of a fundamental frequency $f_{1}$, and several harmonics, as we can see in Fig. 8(a). The first period-doubling bifurcation appears when the temperature difference is increased to $31.4^{\circ} \mathrm{C}$, as shown in Fig. 8(b). In the power spectrum, peaks at $1 / 2 f_{1}$ and its harmonics are produced. The second period-doubling bifurcation occurs when $\triangle T=32.3^{\circ} \mathrm{C}$, producing peaks at $1 / 4 f_{1}$ and its harmonics in the power spectrum, as we can see in Fig. 8(c). The broadband is developing when $\triangle T=34.7^{\circ} \mathrm{C}$ (in Fig. 8(d)), and it is developed when $\triangle T$ is increased to $43^{\circ} \mathrm{C}$ (in Fig. 8(e)). The flow leads to chaos finally. Through analyzing the quasi-periodic route and Feigenbaum route, they are two different methods of increasing frequency peaks to produce the signal broadband, which is gradually developed from finite frequency peaks to infinite.

During the transition to chaos, the thermocapillary convection destabilizes from steady flow to oscillatory flow, and the oscillatory frequency changes with the increase of $M a$. Fig. 9 shows the relationship of oscillatory frequencies with $M a$ for two different experiment conditions. For the silicone oil $1 \mathrm{cSt}$ with the depth of $3 \mathrm{~mm}$, oscillatory frequency varies approximately linearly with $\mathrm{Ma}$, as shown in Fig. 9(a). While for the silicone oil $1.5 \mathrm{cSt}$ with the depth of $2 \mathrm{~mm}$, oscillatory frequency first increases with $\mathrm{Ma}$ gently, and then grows with Ma sharply. The proportional relationship between oscillatory frequency and $M a$ displayed in our experimental study is in agreement with numerical results simulated by Yok-Sheung Li et al. [21,22].

The correlation dimension calculation presented in Fig. 10 reveals that the slope of the linear variation part of the $\ln C(r)-\ln r$ curve becomes independent of $\mathrm{m}$ quickly; it gives $D_{m}=6.86$ at $m=15$ for silicone oil $1 \mathrm{cSt}$, and $D_{m}=10.56$ at $m=23$ for silicone oil $1.5 \mathrm{cSt}$. The corresponding time history of temperature and power spectrum are displayed in Figs. 7 and 8(e). Since the existence of correlation dimension is an important sign to distinguish chaotic signal from random signal, we can prove again that, the thermocapillary flow leads to chaos finally.

\section{Conclusions}

Transition to chaos in thermocapillary convection for different conditions is studied experimentally in the present work. The critical temperature differences of the transition for different fluid depths of silicone oil $0.65 \mathrm{cSt}$ and $1 \mathrm{cSt}$ are collected. The analysis of non-dimensional parameters reveals that the enhancement of buoyant convection and the reduction of aspect ratio can stabilize the flow driven by temperature gradient; this conclusion is consistent with the results by Schwabe [20] and Burguete [9]. Transition for silicone oil $1 \mathrm{cSt}$ with the depth of $3 \mathrm{~mm}$ follows the quasiperiodic route to chaos, and it experiences periodic state, quasiperiodic state, and finally become chaotic. Thermocapillary flow for silicone oil $1.5 \mathrm{cSt}$ with the depth of $2 \mathrm{~mm}$ follows the 
Feigenbaum route, which undergoes 2 period-doubling bifurcations. The proportional relationship between oscillatory frequency and $M a$ obtained in our experimental study is in agreement with numerical results by Yok-Sheung Li et al. [21,22].

\section{Acknowledgment}

This work is supported by the National Nature Science Foundation of China $(10972224,11032011)$ and Knowledge Innovation Program of Chinese Academy of Sciences (KJCX2-YW-L08).

\section{References}

[1] E. Jakeman, D.T.J. Hurle, Thermal oscillations and their effect on solidification processes, Rev. Phys. Technol. 3 (1) (1972) 3-30.

[2] Pierre Berge, Yves Pomeau, Christian Vidal, Order within Chaos: Towards a Deterministic Approach to Turbulence, John Wiley \& Sons, 1984.

[3] M.K. Smith, S.H. Davis, Instabilities of dynamic thermocapillary liquid layers. 1. Convective instabilities, J. Fluid Mech. 132 (7) (1983) 119-144.

[4] M.K. Smith, S.H. Davis, Instabilities of dynamic thermocapillary liquid layers. 2. Surface-wave instabilities, J. Fluid Mech. 132 (7) (1983) 145-162.

[5] P.M. Parmentier, V.C. Regnier, G. Lebon, Buoyant-thermocapillary instabilities in medium-Prandtl-number fluid layers subject to a horizontal temperaturegradient, Int. J. Heat Mass Transfer 36 (9) (1993) 2417-2427.

[6] J.F. Mercier, C. Normand, Buoyant-thermocapillary instabilities of differentially heated liquid layers, Phys. Fluids 8 (6) (1996) 1433-1445.

[7] H.C. Kuhlmann, S. Albensoeder, Three-dimensional flow instabilities in a thermocapillary-driven cavity, Phys. Rev. E 77 (3) (2008) 036303.

[8] R.J. Riley, G.P. Neitzel, Instability of thermocapillary-buoyancy convection in shallow layers Part 1 . Characterization of steady and oscillatory instabilities, J. Fluid Mech. 359 (3) (1998) 143-164.

[9] J. Burguete, N. Mukolobwiez, F. Daviaud, Buoyant-thermocapillary instabilities in extended liquid layers subjected to a horizontal temperature gradient, Phys. Fluids 13 (10) (2001) 2773-2787.
[10] J.P. Gollub, S.V. Benson, Many routes to turbulent convection, J. Fluid Mech. 100 (1980) 449-470.

[11] D. Mukutmoni, K.T. Yang, Rayleigh-Benard convection in a small aspect ratio enclosure 1. Bifurcation to oscillatory convection, J. Heat Transfer - Trans. ASME 115 (2) (1993) 360-366.

[12] D. Mukutmoni, K.T. Yang, Rayleigh-Benard convection in a small aspect ratio enclosure 2. Bifurcation to chaos, J. Heat Transfer - Trans. ASME 115 (2) (1993) 367-376.

[13] Edoardo Bucchignani, Fulvio Stella, Rayleigh-Benard convection in limited domains: Part 2 - Transition to chaos, Numer. Heat Transfer A Appl. 36 (1) (1999) 17-34.

[14] S. Rahal, P. Cerisier, C. Abid, Transition to chaos via the quasi-periodicity and characterization of attractors in confined Benard-Marangoni convection, Eur. Phys. J. B 59 (4) (2007) 509-518.

[15] Edoardo Bucchignani, Daniela Mansutti, Horizontal thermal convection in a shallow cavity: oscillatory regimes and transition to chaos, Int. J. Numer. Methods Heat Flow 10 (2) (2000) 179-195.

[16] Edoardo Bucchignani, Daniela Mansutti, Horizontal thermocapillary convection of succinonitrile: steady state, instabilities, and transition to chaos, Phys. Rev. E 69 (5) (2004) 056319.

[17] Floris Takens, Detecting strange attractors in turbulence, Lect. Notes Math. 898 (1981) 366-381.

[18] Tim Sauer, James A. Yorke, Martin Casdagli, Embedology, J. Stat. Phys. 65 (3/4) (1991) 579-616.

[19] Grassberger Peter, Procaccia Itamar, Measuring the strangeness of strange attractors, Phys. D Nonlinear Phenom. 9 (1/2) (1983) 189-208.

[20] D. Schwabe, A. Scharmann, Measurement of the critical Marangoni number for the transition from stationary to oscillatory thermocapillary convection under microgravitation conditions - results of experiments in Texus 5 and Texus 8 ballistic rockets, Z. Fur Flugw. Weltraumf. 9 (1) (1985) 21-28.

[21] Zhi-Wu Chen, Yok-Sheung Li, Jie-Min Zhan, Double-diffusive Marangoni convection in a rectangular cavity: onset of convection, Phys. Fluids 22 (2010) 034106.

[22] Yok-Sheung Li, Zhi-Wu Chen, Jie-Min Zhan, Double-diffusive Marangoni convection in a rectangular cavity: transition to chaos, Int. J. Heat Mass Transfer 53 (23/24) (2010) 5223-5231. 